\title{
O USO DO COMPUTADOR NA \\ ANÁLISE DE DADOS QUALITATIVOS: \\ QUESTÕES EMERGENTES
}

\section{COMPUTER-AIDED QUALITATIVE DATA ANALYSIS: EMERGING QUESTIONS}

MARIA CAMPOS LAGE

Mestre em Administração de Empresas pelo Centro Universitário Fundação Escola de Comércio Álvares Penteado. Professora pesquisadora da Universidade Presbiteriana Mackenzie (UPM). Av. Dr. Silva Mello, 520, Ed. Rio Negro, ap. 203, Jardim Anhanguera - São Paulo - SP - CEP 04675-010 E-mail: marialage@mackenzie.br

\section{ARILDA SCHMIDT GODOY}

Doutora em Educação pela Universidade de São Paulo (USP).

Professora do Programa de Pós-Graduação em Administração de Empresas da Universidade Presbiteriana Mackenzie (UPM). Alameda dos Aicás, 255, ap. 101, Indianópolis - São Paulo - SP - CEP 04086-000 E-mail: arilda@mackenzie.br 


\section{RESUMO}

A sigla CAQDAS (Computer Aided Qualitative Data Analysis Software) designa softwares criados para auxiliar na análise de dados qualitativos. Alguns autores possuem opiniões favoráveis acerca da utilidade, eficácia e facilidade de uso desses softwares, outros demonstram dúvidas em relação aos benefícios conseguidos, gerando polêmica e tornando oportuna a criação de um espaço para discussão do tema. O objetivo deste trabalho é apresentar e analisar os principais aspectos envolvidos nesse debate, levando em consideração as opiniões e os argumentos "favoráveis" e "contrários" ao uso de CAQDAS. Para atender a esse propósito, foi realizada uma revisão bibliográfica centrada na análise de quatro aspectos: a utilidade do gerenciamento de dados pelo computador, a possibilidade de maior interação do pesquisador com seus dados, a facilitação do processo de comunicação entre pesquisadores e a existência de viés no processo analítico. Discutem-se também algumas experiências nacionais recentes de uso de CAQDAS em pesquisas na área de administração.

\section{PALAVRAS-CHAVE}

Pesquisa qualitativa; Análise de dados; Computador; Software; CAQDAS.

\section{ABSTRACT}

The CAQDAS (Computer Aided Qualitative Data Analysis Software) identifies software packages, created to support qualitative research. Some researches have confidence on software convenience, effectiveness and easiness, but others have concerns about using this kind of tool. There is some discussion regarding computers usage to support qualitative research, establishing an opportune and necessary debate about this subject. The purpose of this essay is to present and analyze the main concerns related to computers usage in qualitative data analysis. Different opinions and arguments are presented, considering the pros and 
cons of CAQDAS, based on a bibliographic review focused in four main points, related to contradictory researchers' opinions: the effectiveness of software data management; the increase of researcher closeness with data; the easiness of researchers' communication process; the possibility of bias in the analytical process. It is discussed too some recent Brazilian experiences in CAQDAS usage, to illustrate the points discussed herein.

\section{KEYWORDS}

Qualitative research; Data analysis, Computer; Software, CAQDAS.

\section{INTRODUÇÃO}

Desde a década de i980, uma ampla variedade de softwares tem sido criada para auxiliar na análise de dados qualitativos. Para referir-se a eles, é comum a utilização da sigla CAQDAS - Computer Aided Qualitative Data Analysis Software. $\mathrm{O}$ uso de tais programas tem sido alvo de intensos debates, envolvendo o que muitos autores classificam como "aplicação da informática na análise de dados".

Existem diversos tipos de CAQDAS, tais como os voltados para gerenciamento de dados, pesquisa de textos, construção de mapas conceituais e construção de teoria com base em atividades de codificação e recuperação. Na literatura recente sobre o tema, as ferramentas mencionadas com mais freqüência são a Atlas.ti, NUD*IST/NVivo e HyperRESEARCH, todas destinadas à construção de teoria com base em atividades de codificação e recuperação de textos (DOLAN; AYLAND, 200I).

O posicionamento dos pesquisadores em relação a esse assunto pode ser identificado por meio de, pelo menos, três grupos: os que preferem técnicas artesanais de trabalho, sem apoio de computador; os que trabalham com programas de computador não desenhados para esse fim, como os processadores de textos e planilhas de cálculo; e aqueles que utilizam pacotes de programas desenvolvidos especificamente para esse tipo de análise (PUEBLA, 2003). O terceiro grupo vem crescendo nos grandes centros de pesquisa, sendo possível encontrar pesquisadores dedicados ao estudo da aplicação de CAQDAS. Por sua vez, os fabricantes das ferramentas têm investido em novas funcionalidades para os pacotes já existentes, gerando um conjunto extenso de opções (JOHNSTON, 2006).

Embora cada aplicativo apresente características específicas, existe uma convergência em torno das principais tarefas de gerenciamento e análise de dados que eles podem realizar. A maioria dos pacotes pode ser descrita como aplicativos para a estocagem, indexação e recuperação de dados. Eles facilitam a aposi- 
ção de códigos a conjuntos de dados e permitem ao pesquisador recuperar todos os segmentos que compartilham um mesmo código. Em programas mais sofisticados, outras tarefas estão disponíveis, como a construção de relacionamentos entre segmentos de dados pertencentes a um mesmo texto ou entre textos diversos, e a elaboração de matrizes com tabulações cruzadas de variáveis, códigos ou dimensões de dados (WEITZMAN, 2000).

Há pouca discordância entre os pesquisadores a respeito da utilidade de CAQDAS. Para Caterall (I996), esta possibilidade libera o pesquisador das suas ferramentas tradicionais, tornando o trabalho mais produtivo, especialmente quando o volume de dados é muito grande.

Outro grupo de autores, como Atherton e Eslmore (2007) e Ryan (2004), defende, no entanto, que, para a maior parte dos projetos, o processo de codificação e análise pode ser executado com o apoio de ferramentas de uso geral, como o Microsoft Word e Excel, ou ainda de gerenciadores de bases de dados, como o Microsoft Access. Já Dembkowski e Lloyd (1995) entendem que o uso do computador permite que o pesquisador esteja em constante interação com seus dados. O acesso ao texto original completo, em vez de fragmentos, é mais rápido e de fácil obtenção. A facilidade de recuperação encoraja a análise exploratória logo cedo, ainda durante o estágio de coleta de dados. Mas Morison e Moir (I998) e Dolan e Ayland (200I) advertem que nem todos os softwares facilitam o retorno ao texto original.

No que se refere à possibilidade da ocorrência de viés durante o processo analítico, vale a pena relembrar o artigo de Agar (I99I), no qual o autor expressa sua preocupação de que os programas acabem por dirigir as pesquisas, já que os pesquisadores projetariam seus estudos de acordo com as metodologias de análise implementadas pelo programa.

Enquanto alguns pesquisadores desenvolveram opiniões bastante favoráveis acerca da utilidade, eficácia e facilidade de uso dos softwares, outro grupo de autores tem demonstrado dúvidas em relação aos benefícios conseguidos, gerando polêmica sobre o assunto e tornando oportuna a criação de um espaço de análise e discussão sobre o tema. Goulding (2002) alerta para esse debate envolvendo o uso de computadores nos processos de análise em pesquisa qualitativa, especialmente quando esse uso envolve algum tipo de CAQDAS.

Com o propósito de contribuir com essa discussão, o objetivo deste trabalho de revisão bibliográfica é apresentar e analisar os principais aspectos envolvidos no debate relacionado à efetividade do uso das ferramentas de computador na pesquisa qualitativa, levando em consideração as opiniões e os argumentos "favoráveis" e "contrários" ao uso desses programas. Segundo Noronha e Ferreira (2000, p. I92), "a revisão desempenha importante papel na transferência de informações entre cientistas e seus pares", evidenciando novas idéias e propor- 
cionando a oportunidade de debates sobre temas específicos. Para ilustrarem-se os aspectos discutidos, utilizam-se exemplos trazidos da aplicação prática de softwares do tipo CAQDAS em alguns estudos realizados no campo da administração de empresas.

O trabalho está centrado na análise de quatro focos de interesse que tem apresentado opiniões contraditórias entre os pesquisadores: a utilidade (ou não) do gerenciamento de dados pelo computador, a possibilidade (ou não) de maior interação do pesquisador com seus dados, a facilitação (ou não) do processo de comunicação entre pesquisadores e a existência (ou não) de viés no processo analítico.

Essa discussão revela-se oportuna, uma vez que vem aumentando a utilização de tais programas sem que haja um exame mais sistemático da sua real contribuição para o pesquisador. Além disso, vários programas de pós-graduação stricto sensu no Brasil apresentam iniciativas relacionadas ao uso de CAQDAS em seus centros de pesquisa, caminhando no mesmo sentido de alguns grandes centros internacionais especializados em pesquisa qualitativa. Assim, é preciso experimentar essas novas possibilidades, testá-las e refletir sobre elas, inclusive trocando experiências com outros pesquisadores que vêm utilizando tais programas e analisando seu potencial, como é o caso de Bandeira-de-Mello (2006).

$\mathrm{O}$ trabalho aqui apresentado está focado nas pesquisas relacionadas com as ferramentas voltadas para a construção de teoria (RICHARDS, I998, 2002; STRAUSS; CORBIN, 2008), por serem aquelas que mais suscitam debates sobre a pertinência ou não de sua utilização.

\section{A UTILIDADE (OU NÃO) DO GERENCIAMENTO DE DADOS PELO COMPUTADOR}

O tratamento e a manipulação dos dados são os pontos fortes das principais ferramentas do tipo CAQDAS, trazendo grande vantagem na forma como os dados podem ser acessados, encontrados e visualizados (BLISMAS; DAINTY, 2003). Segundo Dolan e Ayland (200I), esses softwares oferecem excepcionais facilidades para o armazenamento, o gerenciamento e a manipulação dos dados, permitindo trabalhar com volumes proibitivos no processo manual. Parece haver um consenso de que os CAQDAS permitem colocar ordem e reduzir a ambigüidade dos dados coletados (ATHERTON; ESLMORE, 2007).

As pesquisas realizadas por Dembkowski e Lloyd (I995) mostram que as principais ferramentas possuem recursos poderosos para armazenamento e indexação, que podem ser planejados de acordo com as necessidades da pesquisa. Isso permite ao pesquisador acessar seus dados de forma rápida e precisa, sem se perder em montanhas de papéis ou tabelas. A capacidade dos softwares 
para acessar o dado analisado em seu contexto original, ao mesmo tempo que permite comparar todos os dados abstraídos e gerar novas categorias de informação de forma transparente, propicia maior rigor na demonstração dos resultados obtidos (e da forma como foram obtidos). A facilidade para registrar todos os passos da análise e resultados intermediários abre a possibilidade de verificação do processo executado, a qualquer momento (DOLAN; AYLAND, 200I).

A possibilidade de armazenar como dados codificáveis as teorias que suportam a pesquisa, assim como novos resultados encontrados, facilita a elaboração de correlações complexas entre dados-resultados-aspectos teóricos, permitindo testar idéias e hipóteses.

Ferramentas como o NVivo e Atlas.ti implementam recursos para a visualização dessas correlações como redes ou mapas de idéias, que podem ser armazenados em etapas, de acordo com a evolução da análise (DEMBKOWSKI; LLOYD, I995). Os registros das diferentes etapas facilitam a elaboração dos relatórios de pesquisa, assim como a demonstração das etapas seguidas, para efeitos de avaliação geral do processo.

São encontradas poucas críticas relacionadas aos recursos de armazenamento de dados dos CAQDAS. Nos textos analisados, os autores apontaram como ponto fraco as restrições quanto ao tipo de texto aceito pelos CAQDAS, como em Dembkowski e Lloyd (I995). No entanto, isso está relacionado a um problema já superado pelas principais ferramentas do mercado, que atualmente aceitam textos nos mais diversos tipos, tais como rich text e plain text, entre outros. Bauer e Gaskell (2007) lembram que essas ferramentas agilizam e facilitam o trabalho quando há grandes amostras. No entanto, "um simples aumento do tamanho da amostra não irá implicar, necessariamente, que os achados da pesquisa sejam mais válidos" (BAUER; GASKELL, 2007, p. 408).

\section{A POSSIBILIdAdE (OU NÃO) DE MAIOR INTERAÇÃO DO PESQUISADOR COMI SEUS DADOS}

O simples uso do computador como uma ferramenta de apoio na análise qualitativa leva o pesquisador a estabelecer uma nova relação com seus dados (MUKAMURERA; LACOURSE; COUTURIER, 2006). Os principais softwares possuem facilidades para a codificação dos dados (atribuição de nomes para porções de textos, de acordo com as especificações do pesquisador). Os textos codificados podem ser reagrupados, pesquisados e codificados novamente, facilitando a geração de idéias e novas teorias, num processo de grande proximidade entre $o$ pesquisador e seus dados. A qualquer momento, o pesquisador pode voltar ao tex- 
to original, assim como rever o texto codificado em seu contexto (JOHNSTON, 2006).

Para Blismas e Dainty (2003), o trabalho de codificação é extremamente facilitado pelas principais ferramentas CAQDAS, permitindo a redução do volume de dados a ser analisado. O tempo economizado pela automação de algumas funções pode então ser dedicado à reflexão e ao processo analítico. Johnston (2006) também entende que esses softwares liberam tempo e facilitam o trabalho de análise, em razão das funcionalidades que aproximam o pesquisador dos dados codificados e originais simultaneamente.

As principais ferramentas para construção de teoria possuem funções sofisticadas para pesquisa e recuperação de segmentos de textos, permitindo pesquisar não somente os dados originais, mas também os códigos e outros itens gerados pelo pesquisador. Com base em pesquisas rápidas, o pesquisador pode perceber relações entre partes dos textos e códigos e elaborar correlações entre eles. Essas correlações podem ser usadas para identificar teorias emergentes (DEMBKOWSKI; LLOYD, I995; RICHARDS; MORSE, 2007).

Para Dolan e Ayland (200I), diversos recursos implementados nessas ferramentas estimulam uma grande interação do pesquisador com seus dados, permitindo, a qualquer momento, voltar aos dados originais, sem perder os trabalhos de codificação e correlações realizados. Esse ponto de vista é compartilhado por Gilbert (2002). As facilidades de pesquisa e recuperação estimulariam o pesquisador a interagir com seus dados desde o início do processo de coleta.

Para aproveitar todos os recursos disponíveis, Johnston (2006) entende que é interessante que o CAQDAS seja utilizado desde o momento em que se inicia a investigação, ainda na fase de organização do referencial teórico, considerando os textos relacionados à teoria como um dos tipos de documentos. Ainda segundo Johnston (2006), no passado havia uma preocupação com a possibilidade de o uso de computador afastar o pesquisador de seus dados. No entanto, a prática mostra que o risco é oposto: o uso de um CAQDAS muitas vezes aproxima demasiadamente o pesquisador dos dados, levando-o a exagerar nos processos de codificação, podendo comprometer o tempo dedicado ao esforço analítico.

Deve-se, no entanto, considerar que os principais softwares atuais para apoio à análise de dados qualitativos foram desenhados com base na premissa de que o pesquisador precisa tanto de proximidade quanto de distância de seus dados (RICHARDS, I998; BAZELEY, 2007). A proximidade seria necessária para familiarização com as particularidades dos dados e é provida por meio dos recursos rápidos de pesquisa, recuperação e volta ao contexto original. A distância seria necessária para os processos de abstração e síntese. Os recursos típicos das ferramentas para essa fase seriam os mapas conceituais, as consultas de correlação entre códigos e as facilidades de sumarização dos dados. 
A despeito dos inúmeros aspectos positivos observados pelos pesquisadores quanto à capacidade de CAQDAS em permitir uma grande interação do pesquisador com os dados da pesquisa, existem aspectos negativos que precisam ser considerados. Uma grande desvantagem é que tais programas encorajam estruturas de codificação complexas e detalhadas, gerando, algumas vezes, um excesso de codificação, o que pode levar o pesquisador a ficar, literalmente, atolado em seus dados e distantes do contexto original. Blismas e Dainty (2003) observaram que alguns pesquisadores têm dificuldade para associar um título adequado aos textos codificados e aos nós (um tipo de agrupamento de dados), podendo gerar uma interpretação errônea dos conceitos encontrados. Uma possibilidade citada por Goulding (2002) é a redução do material de campo para apenas dados codificados, levando à perda de contato com fontes importantes para estabelecimento de conceitos e desenvolvimento de teorias. Outro ponto significativo é que, pela facilidade de trabalhar com grandes volumes de dados, alguns usuários tendem a permitir um aumento desnecessário dos dados coletados, levando ao risco do comprometimento da análise em profundidade (DOLAN; AYLAND, 200I).

\section{A FACILITAÇÃO (OU NÃO) DO PROCESŞO DE COMUNICAÇÃO ENTRE PESQUISADORES}

A facilitação (ou não) do processo de comunicação entre pesquisadores é um aspecto pouco abordado nas pesquisas envolvendo o uso de CAQDAS. As principais ferramentas disponíveis trabalham com o conceito de projeto (o Atlas.ti trabalha com o conceito de unidade hermenêutica para os projetos). Cada projeto é formado por um conjunto de dados (transcrições de entrevistas, registros de focus group e de observações de campo, teorias, entre outros) e pode ser acessado por apenas um pesquisador ao mesmo tempo. Os principais aplicativos ainda não trabalham em rede. Assim, dois ou mais pesquisadores não podem acessar o mesmo projeto ao mesmo tempo. Recursos de duplicação resolveriam esse problema, mas exigem algum conhecimento adicional de informática (PUEBLA, 2003; RICHARDS; MORSE, 2007).

Outra importante restrição atual é a impossibilidade de comunicação entre sistemas. Um projeto sendo analisado por uma ferramenta não pode ser exportado para outra (por exemplo, entre Atlas.ti e NVivo7). Normalmente, as equipes de pesquisa trabalham com um mesmo tipo de ferramenta, mas isso pode não ser verdade no caso de projetos que envolvem mais de uma instituição. Uma solução adotada para contornar esse tipo de problema é a criação de diversos projetos iguais, para que cada pesquisador trabalhe de forma independente. Nesse caso, resultados parciais podem ser compartilhados e comparados (por 
meio de recursos simples de colaboração eletrônica), permitindo assim a divisão do trabalho e o enriquecendo os resultados finais (DEMBKOWSKI; LLOYD, I995; RICHARDS; MORSE, 2007). No entanto, é importante estar atento às formas de fazer a fusão entre os elementos do projeto (códigos, nós etc.) no caso de pesquisas que envolvem equipes de pesquisadores, trabalhando em diferentes computadores, mesmo que com o mesmo CAQDAS (PUEBLA, 2003).

A experiência dos autores com a ferramenta NVivo7 tem se mostrado coerente com os aspectos discutidos anteriormente. A implementação recente de análise de dados por uma equipe de pesquisadores dedicados a um projeto único levou ao seu desmembramento em quatro subprojetos, que deverão ser reagrupados ao final das análises individuais. Durante a primeira fase, os pesquisadores podem trabalhar em seus computadores pessoais. No entanto, a partir do momento em que for necessário analisar os dados em um único projeto, todos deverão trabalhar compartilhando o mesmo conjunto de dados.

\section{A ExIStÊncIA (OU NÃO) DE VIÉS NO PROCESSO ANALÍTICO}

A existência (ou não) de viés no processo analítico é provavelmente o aspecto mais debatido entre os pesquisadores. Os problemas relatados muitas vezes não estão relacionados com o CAQDAS em si, mas referem-se à forma como o aplicativo é utilizado. Mesmo os usuários mais entusiastas, algumas vezes, mostram-se reticentes quanto a este aspecto, especialmente porque existem situações nas quais as facilidades dos CAQDAS podem levar ao viés nos processos analíticos.

Dembkowski e Lloyd (I995) argumentam que a facilidade e velocidade obtidas com o uso de um CAQDAS podem levar o pesquisador a gerar um volume de textos codificados muito maior que o necessário, dificultando a tarefa de análise e saindo do foco das questões iniciais de pesquisa. Na mesma linha de argumento Dolan e Ayland (200I) defendem que a grande facilidade para gerar nós e árvores de nós (agrupamentos hierárquicos de conceitos relacionados a partes de dados) estimula o pesquisador a elaborar centenas de estruturas, dificultando os processos analíticos, podendo causar uma superficialidade de análise e o comprometimento do resultado final da pesquisa.

O pesquisador que inicia um trabalho com algum CAQDAS com o mesmo modelo cognitivo com o qual trabalha com ferramentas para dados numéricos (por exemplo, o SPSS) está cometendo um grave erro, já que o dado numérico está dado e a informação qualitativa está em construção (PUEBLA, 2003).

Os processos de análise qualitativa de dados compreendem a geração de conceitos e a construção de tipologias e taxonomias. São estes processos que formam o caminho "duro" da análise qualitativa e que continuam sob a responsabilidade 
do pesquisador, independentemente do tipo de CAQDAS utilizado. Assim, o CAQDAS não assegura por si mesmo o rigor, a validade e/ou a confiabilidade da investigação (ATHERTON; ESLMORE, 2007; PUEBLA, 2003; LIMA, 2005). Ao contrário, o processo de análise, a construção de teorias (caso da grounded theory), os critérios de codificação e ainda a definição do desenho da pesquisa são atribuições exclusivas do pesquisador, independentemente da ferramenta de apoio, mesmo as mais sofisticadas como NVivo7 e Atlas.ti. Segundo Goulding (2002, p. 94), "a construção de teoria é uma atividade mental", e muitos pacotes ainda não estão preparados e direcionados para executar esse tipo de tarefa.

Outro ponto debatido refere-se ao fato de todos os softwares possuírem algum tipo de limitação no tratamento de dados. Em algumas situações, o pesquisador pode ser tentado a remover os dados que não podem ser trabalhados pela ferramenta, podendo perder informações preciosas (DEMBKOWSKI; LLOYD, I995).

Preocupados em comparar os resultados de análises realizadas com e sem o apoio de CAQDAS, Dolan e Ayland (200I) realizaram uma pesquisa na qual o mesmo projeto foi analisado por três equipes. Uma delas utilizou apenas processos manuais, a segunda trabalhou com ferramentas comuns de computador e a terceira trabalhou com o apoio de um dos principais CAQDAS do mercado. Os resultados encontrados pelos pesquisadores que trabalharam sem nenhum apoio de tecnologia consideraram aspectos não percebidos por aqueles que trabalharam com apoio de computador (com e sem CAQDAS). O principal motivo foi o fato de os pesquisadores que trabalharam com processos manuais terem obtido uma melhor visão do perfil dos respondentes, por analisarem todo o texto e não parte dele (como ocorre no processo de codificação). Nesse caso, além de diferentes recursos, este grupo também abordou a análise com outro tipo de metodologia, mais adequada aos processos manuais.

Alguns pesquisadores fazem referência ao uso de CAQDAS como justificativa da metodologia escolhida ou ainda mencionam que "os dados foram analisados pelo CAQDAS". No entanto, indicar a ferramenta escolhida não é a mesma coisa que informar a metodologia utilizada na pesquisa. Como agravante, freqüentemente os CAQDAS são vistos (erroneamente) como frameworks que tornam a análise mais robusta, em vez de ferramentas que apenas apóiam o pesquisador no processo analítico e de gerenciamento dos dados (GILBERT, 2002; BLISMAS; DAINTY, 2003; BAZELEY, 2007). Além disso, muitos usuários se mostram seduzidos pela aparente credibilidade de que o uso do CAQDAS traz para sua investigação (DOLAN; AYLAND, 200I).

Nesse aspecto, a maioria dos autores pesquisados é enfática ao afirmar que o uso de um CAQDAS não substitui a intuição do pesquisador ou sua necessidade de fazer julgamentos. Os principais softwares são desprovidos de capacidade 
analítica. O que eles possuem são recursos avançados para tratamento e consulta dos dados, carecendo da habilidade do pesquisador que deve saber "o que" consultar e "como" interpretar os resultados (BLISMAS; DAINTY, 2003). Isto é, softwares não substituem a necessidade de uso contínuo da intuição do pesquisador (DOLAN; AYLAND, 200I). Nessa discussão, é ilustrativo observar a opinião de Heiner Legiwe, um dos desenvolvedores da ferramenta Atlas.ti, que afirma em um memorando, disponível em Strauss e Corbin (2008, p. 260):

Mais importante para entender a filosofia da interpretação de texto assistida por computador é o fato de que os computadores são absolutamente incapazes de compreender o significado de palavras ou frases. Sua força vem do fato de serem capazes de ajudar com todos os tipos de tarefas de ordenamento, estruturação, recuperação e visualização. Isso significa que é inútil esperar que um computador faça qualquer trabalho analítico, por mais simples que seja. Mas um programa de computador pode ser extremamente útil para criar uma ordem a partir de uma massa de notas de campo, de entrevistas, de códigos, de conceitos e de memorandos; para visualizar a rede de conceitos e relações na teoria emergente; e para manter um acompanhamento sistemático da teoria que se desenvolve, começando com os primeiros dados e sua codificação inicial, documentando todos os passos intermediários e terminando com o relatório final de pesquisa.

Outro aspecto destacado refere-se ao processo de codificação, que é amplamente facilitado. Porém, a atribuição de códigos aos textos é uma tarefa que depende totalmente da intuição e do escrutínio do pesquisador. Puebla (2003) adverte que, dada a facilidade que os computadores oferecem para o exercício da codificação, tudo passa a ser codificado, o que resulta numa possível "fetichização" dos dados que decorre de um entendimento equivocado do papel do computador no processo de análise.

Deve-se considerar ainda que todas as ferramentas do tipo CAQDAS contêm uma teoria implícita de análise qualitativa. Assim, uma das maiores críticas ao uso de CAQDAS seria a tendência de forçar os pesquisadores a utilizar um método particular de análise, de acordo com as características da ferramenta (BLISMAS; DAINTY, 2003). Sobre esse aspecto, Dolan e Ayland (200I) argumentam que esses softwares restringem a abordagem metodológica do processo de análise, levando o pesquisador a trabalhar com os recursos disponíveis na ferramenta, que poderão não ser os mais adequados para determinados tipos de pesquisa. Existe ainda o risco de focar a pesquisa somente nos aspectos que podem ser apoiados pelo computador ou então desenhar a pesquisa para que ela seja aderente aos recursos do software (GOULDING, 2002). 
Um último ponto negativo refere-se ao processo de aprendizagem das ferramentas. É cada vez mais comum a auto-aprendizagem por meio dos tutoriais distribuídos com os pacotes de software. Pela alta qualidade dos tutoriais, existe uma confiança quanto à possibilidade de estes serem suficientes para ensinar não apenas o uso do software, mas também o processo de análise qualitativa. Isso é especialmente grave no caso de novos pesquisadores e estudantes de pós-graduação (JOHNSTON, 2006) e pode levar a uma abordagem "pasteurizada" e padrão. Atherton e Eslmore (2007) lembram que nenhum CAQDAS é neutro, e seus protocolos estão baseados em premissas implícitas sobre análise qualitativa.

Como pode ser observado nessa discussão, o conjunto de aspectos negativos relacionados pelos pesquisadores é significativo, mas existem aqueles que apontam aspectos positivos no que concerne ao processo analítico ou, pelo menos, minimizam as críticas anteriores.

Dembkowski e Lloyd (I995) lembram que a facilidade de codificação/recuperação/ agrupamento dos códigos permite a possibilidade de análises muito mais profundas (e/ou em menor tempo) do que seria possível em trabalhos sem o apoio de um CAQDAS. Os códigos gerados pela ferramenta podem ser incluídos nos relatórios de pesquisa, trazendo transparência para os processos de análise e permitindo a crítica de pares. Da mesma forma, a possibilidade oferecida pelos principais softwares de documentar o "porquê" da escolha de uma denominação para um determinado nó ou texto codificado garante clareza ao processo (BLISMAS; DAINTY, 2003; BANDEIRA-DE-MELLO, 2006).

Blismas e Dainty (2003) argumentam que os problemas no uso dos CAQDAS estão normalmente relacionados com o uso equivocado da ferramenta (em geral relacionado com processos deficitários de treinamento) ou com desconhecimento dos recursos de tecnologia da informação. A ferramenta apenas executa os comandos do pesquisador. Os trabalhos de análise e elaboração de teoria requerem a inteligência humana. Os dados serão agrupados conforme solicitação do usuário, e somente ele pode avaliar se o que obteve está relacionado com o contexto da sua investigação.

Toda pesquisa qualitativa deve ser precedida de uma reflexão sobre as possíveis estratégias disponíveis para tratar a questão de pesquisa e uma justificativa quanto à metodologia escolhida (ATHERTON; ESLMORE, 2007). O uso de um CAQDAS não elimina esta necessidade. Pelo contrário, o pesquisador precisa avaliar se a ferramenta escolhida permite a utilização da metodologia adequada. Puebla (2003) defende que a decisão sobre qual CAQDAS utilizar depende dos objetivos da investigação e também da tradição em que se inscrevem o desenho e o tipo de análise que se pretende realizar. Já Atherton e Eslmore (2007) sugerem que a utilização do CAQDAS pode ser bastante útil quando ele é apropriado 
para a questão de pesquisa e coincide com a metodologia escolhida. Um aspecto recorrente abordado por diversos autores, por meio do uso de um CAQDAS para o desenvolvimento de teses ou outros relatórios de pesquisa, consiste no fato de ser possível disponibilizar para apreciação dos avaliadores não apenas as transcrições de entrevistas, mas também todo o processo evolutivo de análise (BANDEIRA-DE-MELLO; CUNHA, 2003; JOHNSTON, 2006).

Uma das principais contribuições dos CAQDAS é a possibilidade de codificações cada vez mais complexas e detalhadas, acompanhadas de recursos para consultas interativas. Esses dois recursos permitem uma nova abordagem para codificação e análise (RICAHRDS, 2002; BAZELAY, 2007). Tais aspectos também foram vivenciados pelos autores deste ensaio, por meio da ferramenta NViv07. Em um projeto que avaliou as conseqüências da implantação de sistemas integrados de gestão com base em um estudo de caso múltiplo, os dados foram inicialmente analisados por meio de uma abordagem tradicional, apenas com o apoio do Microsoft Word, para transcrição das entrevistas e codificação dos textos. Numa segunda etapa, dois anos após a primeira análise, os dados foram reexaminados com o apoio do NVivo7. Nesta etapa, o volume de códigos gerados pelo pesquisador foi muito maior que na primeira etapa, porém os resultados encontrados foram similares. Em razão do grande volume de textos codificados, o pesquisador ficou motivado a aprofundar a análise e, a partir daí, encontrou aspectos não identificados anteriormente.

\section{ANÁLISE DE DADOS QUALITATIVOS APOIADA POR CAQDAS E POR SOFTWARES DE USO GERAL}

Segundo Johnston (2006), a utilização de um CAQDAS não apresenta benefícios significativos quando existe um pequeno volume de dados. Dependendo do tipo de pesquisa planejada, o apoio de softwares de uso geral pode atender plenamente aos requisitos de análise. A despeito de as ferramentas do tipo CAQDAS existirem há mais de vinte anos, muitos pesquisadores trabalham atualmente somente com o apoio de softwares de uso geral, sendo o mais comum o Microsoft Word, por suas facilidades de edição de textos. Deve ainda ser considerado o fato de este software ser amplamente conhecido e utilizado para a digitação dos documentos de pesquisa e normalmente se encontra instalado nos computadores residenciais e no ambiente de trabalho, não carecendo de investimento adicional. Já os aplicativos voltados para geração de teoria, como o NVivo e Atlas. ti, exigem investimento na compra de licenças, e, segundo Dolan e Ayland (200I), existe uma curva de aprendizagem para seu manuseio que precisa ser considera- 
da. Além disso, os principais produtos do mercado não são compatíveis entre si, o que em geral transforma em problema a escolha do pacote de CAQDAS adequado às necessidades da pesquisa, mesmo para pesquisadores já habituados com esse tipo de software (LEE; FIELDING, I998).

A alternativa de trabalhar apenas com os softwares de uso geral seria, no entanto, vantajosa apenas para projetos com pequeno volume de dados, pois existem restrições que se tornam graves em projetos maiores. O processo de cut/ paste, típico nas análises com o apoio do Microsoft Word, traz dificuldades para identificar respondentes ou ainda para voltar ao texto original. Como o texto é fragmentado e os textos codificados não possuem links com o texto original, dados podem ser perdidos ou desconsiderados (DOLAN; AYLAND, 200I). O processo não encoraja o pensamento criativo, podendo levar o pesquisador a procurar no texto somente as categorias ou os grupos de análise previamente estudados na teoria.

É importante pontuar também que as principais ferramentas especializadas possibilitam a gestão e o registro do progresso do trabalho de análise, o que facilita o processo de teorização por meio dos dados (MUKAMURERA; LACOURSE; COUTURIER, 2006). Alguns aspectos desses softwares, como a capacidade de fazer determinados tipos de codificação de forma automática ou ainda os poderosos recursos para pesquisar a ocorrência de palavras-chave em quaisquer textos armazenados, podem economizar um tempo precioso no andamento da pesquisa (JOHNSTON, 2006). Da mesma forma, pelo fato de mecanizar tarefas tediosas e complicadas na organização dos dados, tais como localizar e copiar segmentos de textos, o computador pode trazer grande eficiência e poupar tempo, segundo Bauer e Gaskell (2007). Porém, Heiner (apud STRAUSS; COURBIN, 2008), em seu memorando sobre ferramentas de apoio à análise de dados qualitativos, enfatiza que um CAQDAS é útil somente se fizer parte da rotina do pesquisador. Isto é, utilizar um CAQDAS esporadicamente para um estudo de pequeno porte pode não valer a pena, considerando-se o tempo envolvido na aprendizagem e eventuais custos envolvidos. E, complementa:

Papel, lápis, tesoura, cola, maços de cartões de indexação e paredes cheias de gráficos coloridos podem lhe dar um toque e um sentimento em relação ao seu trabalho de pesquisa diferente daquele dado pela tela de um computador - do tamanho das mesinhas de um avião - e podem ser mais importantes para inspirar sua criatividade do que qualquer diagrama de computador, por melhor que ele seja (HEINER apud STRAUSS; CORBIN, 2008, p. 26I). 


\section{SUMÁRIO DOS ASPECTOS ANALISADOS E DISCUTIDOS}

O debate sobre os aspectos apresentados e discutidos neste trabalho é bastante intenso, mas existe uma concordância em torno de alguns deles. No Quadro I, que resume os principais pontos encontrados, pode ser verificada uma concentração de pontos "favoráveis" para os itens relacionados ao gerenciamento de dados e à interação do pesquisador com os dados. Por sua vez, os aspectos relacionados com possibilidade de viés no processo analítico e facilidades para comunicação entre pesquisadores possuem mais aspectos "contrários" que "favoráveis".

O Quadro I apresenta o resumo das opiniões "favoráveis" e "contrárias" encontradas na literatura pesquisada para os quatro aspectos analisados. A maioria dos itens relacionados é citada em geral por mais de um autor, alguns deles são recorrentes na literatura, desde as clássicas, como de Richards (I998) e Kelle (I998), até os textos contemporâneos, nacionais e internacionais, como em Goulding (2002), Bandeira-de-Mello (2006) e Richards e Morse (2007). Esses itens estão marcados com asterisco no Quadro I e foram apresentados e discutidos nos tópicos anteriores deste trabalho.

\section{ALGUNS EXEMPLOS ILUSTRATIVOS DA APLICAÇÃO DE CAQDAS EM PESQUISAS BRASILEIRAS NA ÁREA DE ADMINISTRAÇÃO DE EMPRESAS}

No Brasil, as ferramentas do tipo CAQDAS são utilizadas em pesquisas nas áreas de ciências humanas e sociais aplicadas há alguns anos, sendo possível encontrar trabalhos nas áreas de educação, psicologia, saúde e políticas publicas, entre outras. No campo da administração de empresas, a experiência é mais recente, mas já existe um volume significativo de pesquisas qualitativas executadas com o apoio dessas ferramentas. Neste item, são apresentados alguns exemplos de trabalhos recentes. O objetivo não é construir uma revisão abrangente da produção brasileira, mas ilustrar a utilização de CAQDAS em pesquisas na área de administração, fornecendo algumas informações a respeito das estratégias metodológicas que guiaram os autores aqui mencionados. 


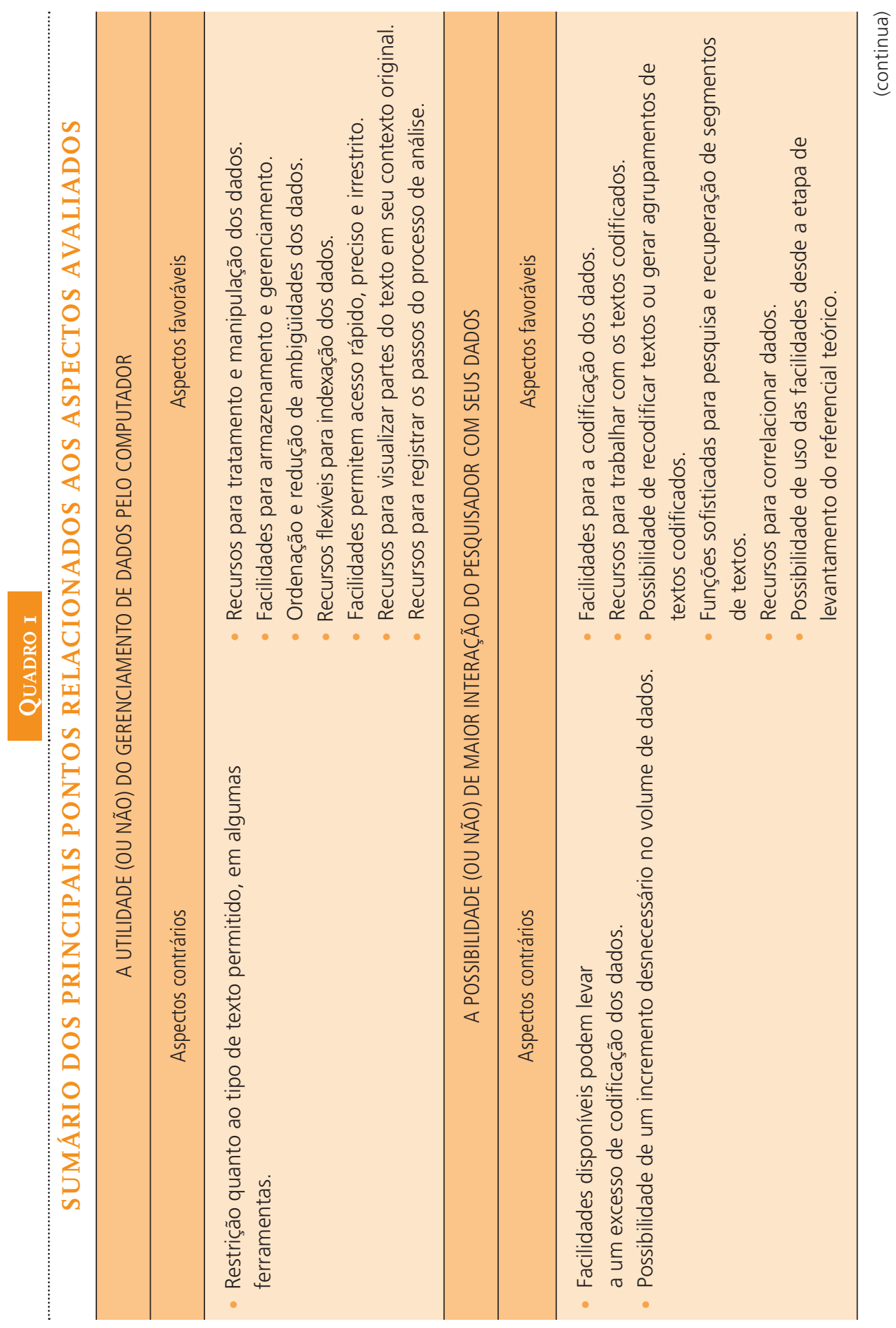




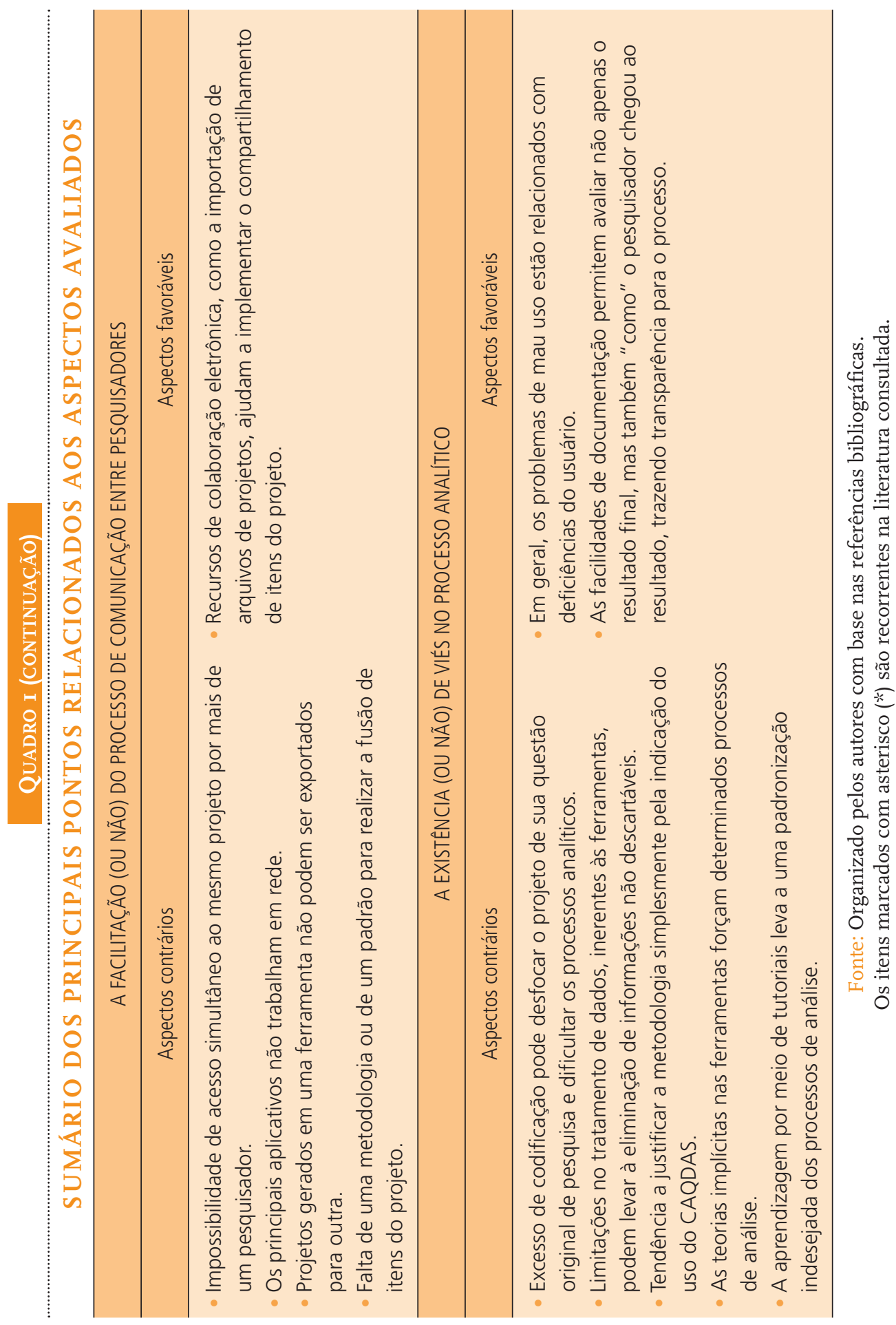


No trabalho de Bacellar, Ikeda e Ângelo (2006), é apresentada uma pesquisa exploratória que investigou a perspectiva de professores de Marketing em relação à atividade de docência, seguindo as orientações metodológicas decorrentes da grounded theory (STRAUSS; CORBIN, 2008). A coleta de dados foi realizada por meio de entrevistas individuais em profundidade, e, para a análise de dados, utilizaram-se os procedimentos da grounded theory. Foram entrevistados dez professores de Marketing com diferentes níveis de experiência (entre I e mais de 20 anos), de ambos os sexos, atuando em faculdades de grande porte do Rio de Janeiro e de São Paulo, tanto na graduação quanto na pós-graduação. O processo de entrevistas seguiu, inicialmente, um roteiro elaborado com base no referencial teórico e na experiência das autoras. Esse roteiro foi alterado conforme as entrevistas e análises iniciais foram realizadas, seguindo assim as orientações de Strauss e Corbin (I998) para o desenvolvimento de pesquisas com base na grounded theory. A coleta de dados gerou i6 horas de gravação e pouco mais de 200 páginas transcritas.

A análise dos dados foi executada com o apoio da ferramenta Atlas.ti versão 5.0, e foram obtidos 953 códigos (agrupamentos de partes dos textos) relacionados ao tema docência no processo de codificação aberta. Os códigos foram distribuídos em oito famílias (agrupamentos): "Início e desenvolvimento da carreira" (286 códigos); "Como vê a aula" (I52 códigos); "Didática e recursos" (I74 códigos); "Por que ser professor" (37 códigos); "Dificuldades de ser professor" (36 códigos); "O que busca para si” (80 códigos); "Como se define” (83 códigos) e "Papel do professor" (I05 códigos). A base teórica utilizada para a interpretação foi o interacionismo simbólico.

$\mathrm{Na}$ apresentação dos resultados, as autoras agruparam os códigos obtidos em cinco grupos: "implementando uma busca: liberdade, refúgio, prazer e status", "percorrendo diferentes caminhos e vencendo obstáculos: nascendo professor e virando professor", "atuando em sala de aula: a composição de um personagem", "atuando em sala de aula: sobre métodos de ensino e recursos didáticos" e "usando a tecnologia em sala de aula: alavancas, muletas e o horror à aula show". Ao longo da apresentação desses grupos, as autoras ilustram as descobertas com trechos das entrevistas.

Dado o tipo de pesquisa, não são apresentadas redes de relacionamento entre os códigos ou qualquer outro recurso gráfico da ferramenta Atlas.ti. Para o leitor, fica apenas a informação da utilização da ferramenta, sem nenhuma citação sobre vantagem ou desvantagem em sua aplicação no processo de codificação. Em termos de metodologia, as autoras enfatizam a explicação das etapas seguidas no processo de coleta de dados e análise dos códigos segundo as etapas da grounded theory. Isso coloca o Atlas.ti em seu devido lugar no processo da pesquisa: apenas uma ferramenta de apoio (BAUER; GASKELL, 2007). 
Outro exemplo ilustrativo refere-se à pesquisa de Bandeira-de-Mello e Cunha (2004) a respeito da adaptação estratégica de pequenas empresas em cenário de turbulência. Nessa pesquisa, um estudo de caso simples, os autores também utilizaram os procedimentos metodológicos da grounded theory. O objetivo foi gerar uma teoria substantiva para explicar o comportamento estratégico de pequenas empresas em contexto de turbulência ambiental causado, principalmente, pela influência governamental.

O objeto de estudo foi uma construtora de edificações de administração familiar, que atua no município de Florianópolis. Foram utilizados dois grupos de dados: primários, com base em nove entrevistas não estruturadas em profundidade com cinco pessoas que atuam ou atuaram como tomadores de decisão na empresa; e secundários, envolvendo informações sobre a história da empresa, resultados financeiros, indicadores e reportagens. Houve triangulação entre os dados primários e secundários.

As entrevistas resultaram em 595 minutos de diálogos que foram transcritos para análise, que contou com o apoio da ferramenta Atlas.ti (os autores não citam a versão do software). Durante a apresentação dos aspectos metodológicos da pesquisa, os autores demonstram ter avaliado os eventuais problemas relacionados ao uso da ferramenta e adotado procedimentos para garantir a qualidade da análise:

Foram tomadas as devidas precauções para evitar os problemas decorrentes do uso de softwares no auxílio ao tratamento interpretativo de dados. Entre eles está a alienação do pesquisador e a conseqüente reificação dos códigos, o que torna a análise mecânica e distanciada da essência dos dados (BANDEIRA-DE-MELLO; CUNHA, 2004, p. I63).

A codificação dos dados seguiu os procedimentos previstos na metodologia, que divide o processo em três fases não-lineares (codificação aberta, axial e seletiva). Os autores consideraram os dados secundários nos processos de análise como subsídios à sensibilização e como referenciais para identificar divergências entre os dados objetivos e as percepções dos entrevistados. Foram utilizadas 678 citações, I77 códigos e 50 notas de análise. Do total de códigos, I37 são de primeira ordem, diretamente ligados às citações, e 40 são constructos teóricos abstratos.

Além do processo de codificação, os autores citam outras duas atividades que foram apoiadas pela ferramenta Atlas.ti: a) o ataque à teoria por meio do recurso de query da ferramenta, como uma das formas de aprimorar a qualidade da teoria em cada um dos critérios perseguidos pelo design da pesquisa; b) a análise dos relatórios gerados pelo Atlas.ti para auditorias do processo de pesquisa. 
$\mathrm{Na}$ apresentação dos resultados, os autores discorrem sobre as etapas para geração da teoria substantiva, ilustrando os pontos significativos com trechos das falas dos entrevistados. Quanto ao uso da ferramenta, também nesse exemplo, os autores se limitam a citar as principais atividades do processo de análise que foram apoiadas pelo Atlas.ti, mantendo o software como coadjuvante do processo como um todo. Os pesquisadores, os pesquisados e principalmente os dados são os atores principais no relatório analisado.

No exame desses exemplos, é importante chamar atenção para o fato de que, a despeito de a utilização de ferramentas do tipo CAQDAS estar freqüentemente associada à grounded theory (que em português foi traduzida para teoria fundamentada), os principais softwares, como NVivo7 e Atlas.ti, podem ser aplicados a qualquer tipo de pesquisa qualitativa que trabalhe com texto, imagem ou som, desde que sejam respeitados eventuais limites tecnológicos, inerentes à ferramenta em questão. Isso pode ser observado nos exemplos apresentados a seguir.

Um deles é o estudo de Pascotto e Machado (2006), que pesquisa as dificuldades enfrentadas pelas pequenas empresas durante seus primeiros anos de vida. A pesquisa classificada como "do tipo interpretativa, de corte transversal" (PASCOTTO; MACHADO, 2006, p. 59) envolveu um estudo de caso múltiplo, que trabalhou com empresas prestadoras de serviços de seguros, na fase inicial de desenvolvimento, localizadas no Paraná. O objetivo foi identificar os problemas enfrentados pela pequena empresa do setor de serviços securitários nos primeiros anos de atividade.

Os dados primários foram coletados por meio de entrevistas individuais semi-estruturadas e pesquisa documental, que se limitou à análise do contrato social e dos demonstrativos financeiros das empresas. Esses dados foram analisados com o auxílio do software NVivo versão I.I. Os pesquisadores trabalharam com análise de conteúdo para interpretação dos dados, que foram inicialmente codificados com o auxílio do NVivo.

Com base na análise de conteúdo, os autores apresentam os resultados obtidos, ilustrados por trechos das entrevistas. Ao final, é construído um quadro resumo das dificuldades apresentadas pelos empreendedores de serviços securitários. Como nos estudos anteriores, os autores não fazem apologia do uso da ferramenta, tampouco apresentam alguma restrição relacionada ao uso do software.

Um último exemplo a ser comentado é a dissertação de Zoschke (2006) que apresenta um estudo multicaso exploratório do marketing empreendedor e suas ligações com a rede de relação dos empreendedores de duas empresas.

As entrevistas foram semi-estruturadas em profundidade e utilizou-se um roteiro flexível, que permitia alterações, se necessário. Ao final do processo de coleta de dados, as entrevistas foram convertidas para $\mathrm{MP}_{3}$ e analisadas com 
apoio do software Atlas.ti. Utilizaram-se ainda dados secundários, compostos por fotos, artigos de jornais e informativos impressos das empresas participantes da pesquisa.

Os dados foram analisados por meio da codificação, de descrições e comparações de elementos relativos à rede de relação e ao marketing empreendedor nas empresas pesquisadas. Para "estruturar e facilitar a descrição e a análise dos dados" (ZOSCHKE, 2006, p. 57), foi utilizada a análise de períodos temporais, que descreve os processos que compõem o fenômeno estudado segundo sua seqüência temporal e sua integração no contexto das empresas estudadas.

$\mathrm{Na}$ apresentação dos dados, a autora discorre sobre os resultados obtidos, exemplificando os diversos períodos e fatos encontrados, com fragmentos dos textos. Ao final, é elaborado um quadro sumário, que apresenta uma seqüência de fatos significativos e uma explicação para cada quadro, por meio de extratos das entrevistas. Segundo Zoschke (2006, p. 62): "Os elementos de destaque indicados nos diferentes quadros [...] são códigos que se mostraram importantes na análise de dados com o uso do software Atlas.ti."

Nesse estudo, a autora toma o cuidado de esclarecer cada etapa da investigação e em quais momentos foram utilizados os resultados obtidos nas codificações e estruturações com a ferramenta de apoio, deixando claro o papel desta na pesquisa como um todo.

\section{CONSIDERAÇÕES FINAIS}

Existe um consenso entre os autores, mesmo aqueles mais reticentes quanto ao uso de CAQDAS, sobre a eficiência desse tipo de aplicativo nos processos de gerenciamento e recuperação dos dados qualitativos. As experiências relacionadas nos artigos analisados demonstram que, especialmente no caso de grandes volumes de dados, esses processos são amplamente facilitados por meio de ferramentas especialistas. As facilidades para registro detalhado das etapas seguidas pelo pesquisador no processo de análise trazem transparência ao processo.

Para que tudo isso funcione, é necessário que o pesquisador escolha registrar as etapas seguidas. A ferramenta não toma esse tipo de decisão, aspecto para o qual existe consenso. Os softwares não prescindem da inteligência, intuição e criatividade do pesquisador, pois é ele quem vai direcionar os rumos da pesquisa, escolher os melhores recursos, decidir quando deve parar de codificar dados e aprofundar o processo analítico, assim como decidir quando a análise está concluída. 
As ferramentas do tipo CAQDAS são apenas, como o próprio nome diz, ferramentas de apoio. Quem analisa é o pesquisador. No entanto, as práticas adotadas para a formação de novos pesquisadores tendem a deturpar esse conceito. Torna-se necessário reavaliar esses processos, talvez incluindo a análise com apoio de CAQDAS como parte integral da formação, como recomendado por Johnston (2006), e não apenas dedicando a eles uma palestra no final do curso.

É importante observar que a maioria dos artigos brasileiros que discutem o uso de ferramentas do tipo CAQDAS faz referências a versões antigas dos softwares. Por exemplo, muitos textos atuais citam a ferramenta Nud*ist ou NVivo2 em comparativos com outras ferramentas. Esses softwares foram substituídos no final de 2006 pelo software NVivo7, que corrige deficiências importantes nos softwares anteriores (como limitações de tipo de arquivo texto ou ainda a possibilidade codificação de imagens). Os principais fornecedores de CAQDAS lançaram novas versões de seus produtos no final de 2006 e já anunciam novidades para 2008 , algumas delas resolvendo questões críticas, como o trabalho em equipe.

As novas gerações de ferramentas em geral estão totalmente integradas aos aplicativos Microsoft Windows, permitindo novas possibilidades para atividades inerentes ao processo de pesquisa. Um aspecto enfatizado é a possibilidade de compartilhamento das análises e interpretações entre grupos de pesquisadores que trabalham com o mesmo tipo de ferramenta. Os arquivos de dados de um projeto (como transcrições, textos codificados e matrizes comparativas) podem ser "salvos" e enviados através de e-mails, para serem "carregados" na base de dados de um outro computador, permitindo a troca de experiências entre pesquisadores ou ainda entre um orientado e seu orientador (RETTIE et al., 2008).

A despeito das facilidades obtidas com os novos recursos, o software não pode ser visto como o senhor da pesquisa, conforme evidenciado nos exemplos apresentados neste trabalho. Os pesquisadores são os atores do processo. A ferramenta é um recurso que pode, se bem utilizado, facilitar o gerenciamento dos arquivos, agilizar a codificação e busca de respostas, além de facilitar a comunicação. Mas quem decide o que fazer, quando fazer e por que fazer continua sendo o pesquisador.

\section{REFERẾNCIAS}

AGAR, M. H. The right brain strikes back. In: FIELDING, N. G.; LEE, R. M. (Ed.). Using computers in qualitative research. London: Sage, I99I.

ATHERTON, A.; ESLMORE, P. Structuring qualitative enquiry in management and organization research: a dialoge on the merits of using software for qualitative data analysis. Qualitative Research in Organizations and Management: An International Journal, v. 2, n. I, p. 62-77, 2007. 
BACELLAR, F. C. T.; IKEDA, A. A.; ÂNGELO, M. Professores de marketing e a docência em ensino superior: revelando trajetórias e compreendendo perspectivas. In: ENCONTRO NACIONAL DA ASSOCIACAO NACIONAL DE PÓS-GRADUAÇÃO EM ADMINISTRAÇÃO, 30., 2006, Salvador. Anais... Salvador: Anpad, 2006. I CD-ROM.

BANDEIRA-DE-MELLO, R. Operacionalizando o método da grounded theory nas pesquisas em estratégia: técnicas e procedimentos de análise com apoio do software Atlas.ti. In: ENCONTRO DE ESTUDOS EM ESTRATÉGIA DA ANPAD, I., 2003, Curitiba. Anais... Curitiba: Anpad, 2003. I CD-ROM. Softwares em pesquisa qualitativa. In: SILVA, A. B. da; GODOI, C. K.; BANDEIRA-DEMELLO, R. (Org.). Pesquisa qualitativa em estudos organizacionais: paradigmas, estratégias e métodos. São Paulo: Saraiva, 2006.

BANDEIRA-DE-MELLO, R.; CUNHA, C. J. C. A. Administrando risco: uma teoria substantiva da adaptação estratégica de pequenas empresas a ambientes turbulentos e com forte influência governamental. Revista de Administração Contemporânea, Edição Especial, p. I57-I79, 2003.

BAUER, M. W.; GASKELL, G. Pesquisa qualitativa com texto, imagem e som: um manual prático. Rio de Janeiro: Vozes, 2007.

BAZELEY, P. Qualitative Data Analysis with NVivo. London: Sage, 2007.

BLISMAS, N.; DAINTY, A. Computer-aided qualitative data analysis: panacea or paradox? Building Research \& Information, v. 3I, n. 6, p. 455-463, Nov. 2003.

CATERALL, M. Using computer programs to code qualitative data. Marketing Intelligence and Planning, v. I4, n. 4, p. 29-33, I996.

DEMBKOWSKI, S.; LLOYD, S. H. Computer applications - a new road of qualitative data analysis? European Journal of Marketing, v. 29, n. II, p. 27-37, I995.

DOLAN, A.; AYLAND, C. Analysis on trial. International Journal of Market Research, v. 43, quarter 4, p. 377-389, 200 I.

GILBERT, L. S. Going the distance: "closeness" in qualitative data analysis software. International Journal of Social Research Methodology, v. 5, n. 3, p. 215-228, 2002.

GOULDING, C. Grounded theory: a practical guide for management, business and market researchers. London: Sage, 2002.

JOHNSTON, L. Software and method: reflections on teaching and using QSR NVivo in doctoral research. International Journal of Social Research Methodology, v. 9, n. 5, p. 379-39I, Dec. 2006.

KELLE, U. Introduction: an overview of computer-aided methods in qualitative research. In: (Org.). Computer-aided Qualitative Data Analysis: theory, methods and practices. London: Sage, 1998.

LEE, R. M.; FIELDING, N. G. Users'experiences of qualitative data analysis software. In: KELLE, U. (Org.). Computer-aided Qualitative Data Analysis: theory, methods and practices. London: Sage, I998.

LIMA, E. O. Métodos qualitativos em administração: teorizando a partir de dados sobre processos em uma recente pesquisa. In: ENCONTRO NACIONAL DA ASSOCIAÇÃO NACIONAL DE PÓS-GRADUAÇÃO EM ADMINISTRAÇÃO, 29., 2005, Brasília. Anais... Brasília: Anpad, 2005. I CD-ROM.

MORISON, M.; MOIR, J. The role of computer software in the analysis of qualitative data: efficient clerk, research assistent or Trojan horse? Journal of Advanced Nursing, v. 28, n. I, p. Io6-II6, I998. 
MUKAMURERA, J.; LACOURSE, F. COUTURIER, Y. Des avancées en analyse qualitative: pour une transparence et une systématisation des pratiques. Recherches Qualitatives, v. 26, n. I, p. IIoI38, 2006.

NORONHA, D. P.; FERREIRA, S. M. P. Revisões de literatura. In: CAMPELLO, B. S.; CENDÓN, B. V.; KREMER, J. M. (Org.). Fontes de informação para pesquisadores e profissionais. Belo Horizonte: Editora da UFMG, 2000. p. I9I-I98.

PASCOTTO, H.; MACHADO, H. P. V. Dificuldades de pequenas empresas do setor de serviços securitários nos primeiros anos de vida. Gestão.Org - Revista Eletrônica de Gestão Organizacional, v. 4, n. I, jan./abr. 2006. Disponível em: <www.gestaoorg.dca.ufpe.br>. Acesso em: 8 fev. 2008. PUEBLA, C. A. C. Análisis cualitativo asistido por computadora. Sociologias, n. 9, p. 288-313, ene./ jun. 2003.

RETTIE, R. et al. CAQDAS: a supplementary tool for qualitative market research. Qualitative Market Research: An International Journal. v. II, n. I, p. 76-88, 2008.

RICHARDS, L. Closeness to data: the changing goals of qualitative data handling. Qualitative Health Research, v. 8, n. 3, p. 319-328, 1998.

Qualitative Computing - a methods revolution? International Journal of Social Research Methodology, n. 5, v. 3, p. 263-276, 2002.

RICHARDS, L.; MORSE, J. Readme first for a user's guide to qualitative methods. 2. ed. Thousand Oaks: Sage, 2007.

RYAN, G. W. Using a Word processor to tag and retrieve blocks of text. Field Methods, v. I6, n. I, p. IO9-I30, 2004 .

STRAUSS, A.; CORBIN, J. Pesquisa qualitativa: técnicas e procedimentos para o desenvolvimento de teoria fundamentada. 2. ed. Porto Alegre: Artmed, 2008.

WEITZMAN, E. A. Software and qualitative research. In: DENZIN, N. K.; LINCOLN, Y. S. (Ed.). Handbook of qualitative research. 2. ed. Thousand Oaks: Sage, 2000. p. 803-820.

ZOSCHKE, A. C. K. Marketing empreendedor e rede de relação: um estudo multi-caso de micro, pequenas e médias empresas tecnológicas de Blumenau. 2006. Dissertação (Mestrado em Administração)Faculdade de Administração, Universidade Regional de Blumenau, Blumenau, 2006.

\section{TRAMITAÇÃO}

Recebido em 15/2/2008

Aprovado em 31/3/2008 